\title{
Anabolic Steroid-induced Reversible Cardiomyopathy in a Young Non-athletic Female
}

\author{
Uzma Gul and Muhammad Shahid \\ Department of Cardiology, Russells Hall Hospital, NHS Trust, Dudley, United Kingdom
}

\begin{abstract}
Anabolic androgenic steroid (AAS) abuse is widespread nowadays, not only in athletes but in non-athletic populations; and rapidly becoming a public health challenge. Cardiomyopathy (both dilated and hypertrophic) is a known complication of anabolic steroid use. A 47-year woman presented with acute pulmonary edema, in the background of exertional dyspnea for the last few weeks. Echocardiogram revealed severe left ventricular systolic dysfunction in the presence of hypertrophy, global hypokinesia, and septal dyssynchrony. She was treated with guideline-directed treatment for heart failure. Investigation into the causes revealed that she had been using anabolic of steroids for social reasons. She was counselled and managed to abstain from the use steroids. Cardiac MRI, five months later, showed mild left ventricular systolic impairment and no hypertrophy. There was no perfusion defect, scar or infiltration. At nine months follow-up, she improved symptomatically with better exercise tolerance.
\end{abstract}

Key Words: Anabolic steroids, Cardiac MRI, Cardiomyopathy.

How to cite this article: Gul U, Shahid M. Anabolic Steroid-induced Reversible Cardiomyopathy in a Young Non-athletic Female. J Coll Physicians Surg Pak 2022; 32(02):233-235.

\section{INTRODUCTION}

Anabolic androgenic steroid (AAS) abuse is widespread nowadays and rapidly becoming a public health concern. ${ }^{1}$ The lifetime prevalence of AAS among male adolescents in Western countries is $5 \%$ to $10 \% .{ }^{1}$ Emergence of websites selling synthetic AAS with misleading information and lack of strict regulations have contributed to the problem. ${ }^{2}$ Despite education/awareness about the potential side effects, many users will continue their practice to alter appearance or augment performance. ${ }^{3} \mathrm{AAS}$ use has shifted from the elite athlete to the average population for fitness or appearance and role change. ${ }^{1}$ Cardiomyopathy (both dilated and hypertrophic) is a known complication of anabolic steroid use. ${ }^{4}$

We, herein, present a case of a young adult female presenting with acute pulmonary edema, secondary to acute left ventricular failure and secondary to AAS abuse. She improved symptomatically on abstinence from AAS and symptomatic treatment of heartfailure.

Correspondence to: Dr. Uzma Gul, Department of Cardiology, Russells Hall Hospital, NHS Trust, Dudley, United Kingdom

E-mail: uzmagulcardiology@gmail.com

Received: December 19, 2019; Revised: April 20, 2020;

Accepted: April 26, 2020

DOI: https://doi.org/10.29271/jcpsp.2022.02.233

\section{CASE REPORT}

A 47-year woman presented to Emergency Department with worsening breathlessness and cough with pink frothy sputum, in the background of exertional shortness of breath for a few weeks. Examination showed distended jugular veins, bilateral crackles, and peripheral edema. Electrocardiogram showed sinus rhythm with left bundle branch block. Chest X-ray showed features of evolving pulmonary edema. NT-proBNP levels were raised at 1,561 $\mathrm{ng} / \mathrm{L}$ and rest of the biochemical profile was normal. She was admitted to high dependency unit (HDU) and treated for acute heart failure and community acquired pneumonia. Past history was significant for non-Hodgkin lymphoma 20 years back that was successfully treated with chemo and radiotherapy. She was ex-smoker, normotensive and normoglycemic. She admitted taking testosterone over past several months to change appearance and role, but denied high alcohol intake or illicit drugs use. There was no family history of cardiomyopathy or sudden cardiac death, although positive family history of premature ischemic heart disease was present. Inpatient echocardiogram revealed left ventricular (LV) hypertrophy and severe global systolic dysfunction. She responded to treatment and was discharged with planned follow-up. She was advised to refrain from testosterone, which she agreed and was compliant to the advice as assessed on follow-up.

Repeat transthoracic echo performed three months later showed global hypokinesia and septal dyssynchrony with severely impaired LV systolic function, Simpson's biplane ejection fraction of $34 \%$ and normal right heart. She was treated 
with guideline-directed medical therapy including maximum tolerated doses of beta blocker, angiotensin converting enzyme inhibitor, and aldosterone antagonist.

Investigations for underlying etiology of cardiomyopathy did not show folic acid or vitamin B12 deficiency. Her autoimmune screening including anti-nuclear antibody (ANA) and rheumatoid factor were negative. Iron studies were normal, as were thyroid function tests. There was no evidence of recurrence of lymphoma. Pheochromocytoma, celiac and myeloma screen were negative. CT coronary angiography ruled out coronary artery disease.

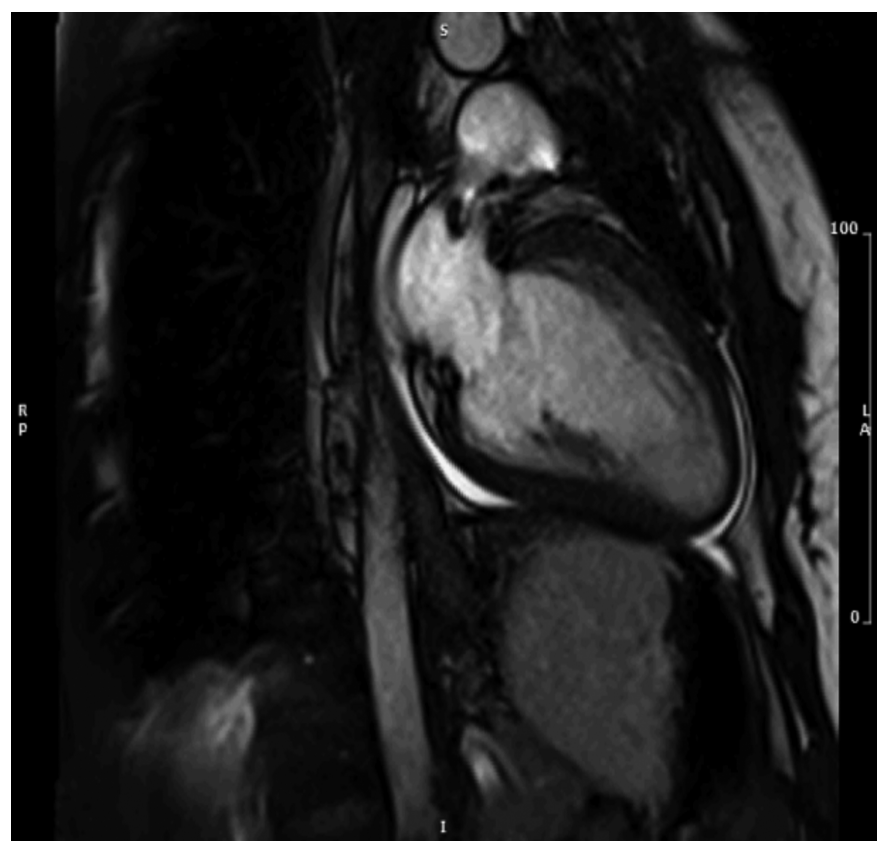

Figure 1: Cardiac MRI - End diastolic frame from two chamber cine (bSSFP).

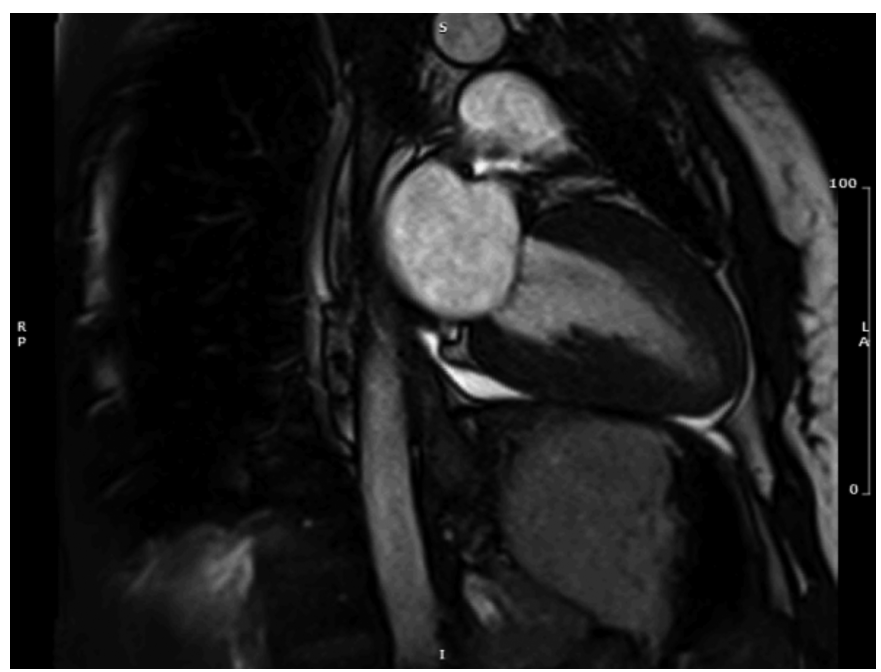

Figure 2: Cardiac MRI-End systolic frame from two chamber cine (bSSFP).

Cardiac MRI with vasodilator stress and gadolinium was performed five months after initial admission. Cine images showed normal sized, non-hypertrophied LV with discordant septal motion, but with no other regional motion abnormality; mildly impaired systolic function with ejection fraction of $57 \%$
(Figures 1 and 2). First pass perfusion with adenosine achieved adequate response. There were neither signs of inducible ischemia in any coronary territory nor microvascular dysfunction. Tissue characterisation showed no evidence of myocardial infiltration, inflammation or scar (Figure 3). Extracardiac findings were well developed muscles and near absence of breasttissue.

Nine months later, she was symptomatically much better and had improved exercise tolerance.

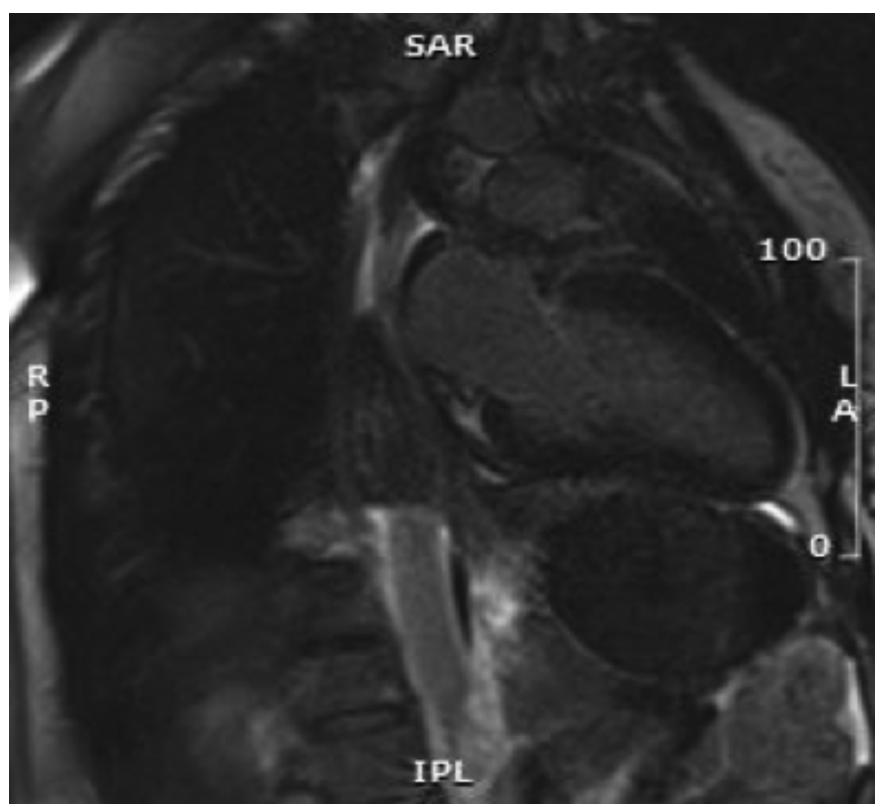

Figure 3: Cardiac MRI - Late gadolinium (PSIR) image shows no enhancement.

\section{DISCUSSION}

Use of supraphysiological testosterone dose results in increased muscle mass and strength, but this comes at a cost. ${ }^{4}$ Cardiomyopathy (both dilated and hypertrophic) is a known complication of anabolic steroid use. ${ }^{4}$ In addition, it is associated with changes in appearance, gonadal function and psychiatric disturbance.

AASs cause direct cardiac toxicity through receptors expressed on myocytes, as well as exaggerated LV hypertrophic response to any pressure overload state. ${ }^{1,3}$ In addition, exposure to AAS is associated with increase sympathetic tone and inflammatory markers. Other effects include hypertension, dyslipidemia, arrhythmias and sudden death. ${ }^{5}$

Adverse effects of AAS abuse in athletes have been reported. Our case is the first to report AAS-induced cardiomyopathy in a non-athleticfemale.

Reversal of myocardial effects after discontinuation is controversial. ${ }^{3-6}$ One study showed that although systolic dysfunction may be reversible, diastolic dysfunction was persistent. ${ }^{3}$ Residual hypertrophy has also been reported. ${ }^{3}$ Athletes have been shown to have persistent LV hypertrophy many years after discontinuation of AAS abuse. ${ }^{3}$ Whereas, partial recovery of function and reversal of increased LV mass has been reported by few case reports. ${ }^{3}$ Nieminen and colleagues described two AAS users 
presenting with dilated cardiomyopathy and in one systolic function returned to normal nine months after discontinuation.

Factors such as duration of AAS abuse, and presence or absence of athletic training may contribute to the varied spectrum of long-term outcome. Our case highlights rapid and near complete recovery and no signs of permanent damage on cardiac MRI with early abstinence. Whereas some reports showed severe hypertrophy and myocardial scarring on cardiac MRI after long lasting abuse of AAS. ${ }^{2}$

Since LVhypertrophy is an independent risk factorforcardiovascular mortality, AAS use potentially increases death risk. ${ }^{3}$ Whether reversal of hypertrophy translates into decrease in mortality risk needs to be proven and may depend upon whether permanent damage (fibrosis) has occurred or not.

There is currently no diagnostic gold standard for this condition, and the temporal sequence of events and the absence of alternative aetiologies may support diagnosis. Early detection (before irreversible damage) and AAS abstinence along with traditional heartfailuretreatment have been shown to beeffective. Prevention of this form of cardiomyopathy, however, may be achievable. Formulating strict drug regulations and promoting public awareness may help preventAAS use.

\section{PATIENT'S CONSENT:}

Written informed consent was obtained.

\section{CONFLICT OF INTEREST:}

The authors declared no conflict of interest.

\section{AUTHORS' CONTRIBUTION:}

UG: Writing, reviewing and drafting the manuscript, extracting still frames \& cine.

MS: Proofreading the manuscript and extracting the images.

\section{REFERENCES}

1. Flo FJ, Kanu O, Teleb M, Chen Y, Siddiqui T. Anabolic androgenic steroid-induced acute myocardial infarction with multiorgan failure. Proc (BaylUniv Med Cent) 2018; 31(3):334-6. doi: 10.1080/08998280.2018.1460130.

2. Gangadharamurthy D, Pandian N, Malhotra S, Tahir S. Anabolic androgenic steroid abuse and reversible cardiomyopathy: An emerging and under-recognised cardiovascular public health problem among fitness enthusiasts. J Am Coll Cardiol 2018; 71(11):A2426. doi: 10.1016/S0735-1097(18)32967-X.

3. Ha ET, Weinrauch ML, Brensilver J. Non-ischemic cardiomyopathy secondary to left ventricular hypertrophy due to long-term anabolic-androgenic steroid use in a former olympic athlete. Cureus 2018; 10(9):e3313. doi:10.7759/cureus.3313.

4. Garner O, lardino A, Ramirez A, Yakoby M. Cardiomyopathy induced by anabolic-androgenic steroid abuse. BMJ Case Rep 2018; 2018: bcr-2017-223891. doi: 10.1136/bcr-2017223891.

5. Joseph J, Naqvi SY, Sturm E. Reversible anabolic androgenic steroid-induced cardiomyopathy. Cardiovasc Dis Med 2017; 2(3):1-3.doi: 10.15761/CDM.1000131.

6. Li C, Adhikari BK, Gao L, Zhang S, Liu Q, Wang Y, et al. Performance-enhancing drugs abuse caused cardiomyopathy and acute hepatic injury in a young bodybuilder. Am J Mens Health 2018; 12(5):1700-4. doi: $10.1177 / 1557988318783504$.

7. Nieminen MS, Ramo MP, Viitasalo M, Heikkila P, Karjalainen J, Mantysaari $M$, et al. Serious cardiovascular side effects of large doses of anabolic steroids in weight lifters. Eur Heart J 1996; 17(10):1576-83. doi: 10.1093/oxfordjournals. eurheartj.a014724. 